\title{
Annealing effect on thermoluminescence (TL) glow curve of $\mathrm{Ca}_{0.99} \mathrm{SO}_{4}: \mathrm{Eu}_{0.005} \mathrm{Dy}_{0.005}$ phosphor
}

\author{
Y. Rangeela Devi ${ }^{1 *}$, S. Dorendrajit Sing ${ }^{2}$ \\ ${ }^{\mathrm{I}}$ Department of Physics, Pachhunga University College, Aizawl 7960oI, India \\ ${ }^{2}$ Department of Physics, Manipur University, Imphal 795003, India
}

\begin{abstract}
$\mathrm{Ca}_{1-\mathrm{x}-\mathrm{y}} \mathrm{SO}_{4}: \mathrm{Eu}_{\mathrm{x}} \mathrm{Dyy}_{\mathrm{y}}(0<\mathrm{x}<0.04,0<\mathrm{y}<0.04)$ phosphors were prepared and characterized by XRD. The samples were well crystalline and exhibit orthorhombic structure. The phosphor $\mathrm{Ca}_{0.99} \mathrm{SO}_{4}: \mathrm{Eu}_{0.005} \mathrm{Dy}_{0.005}$ was found having highest TL intensity. The phosphor was annealed at different temperatures. All the TL glow curves have one prominent peak at 420K. TL intensity for this peak was found decreasing when the phosphor was annealed at a particular temperature for different durations.
\end{abstract}

Key words: Thermoluminescence, $\mathrm{XRD}$, annealing, sensitivity.

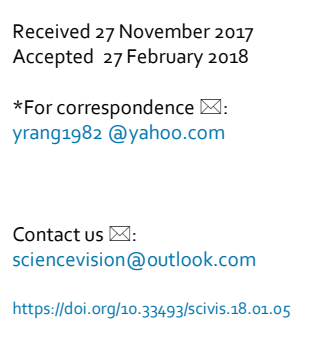

Received 27 November 2017
Accepted 27 February 2018

*For correspondence $\triangle$ : yrang1982@yahoo.com

Contact us $\square$ :

https://doi.org/10.33493/scivis.18.01.05

\section{Introduction}

Amongst TL phosphors, sulphates have been very popular for use in radiation dosimetry. Sulphate-based radiation dosimetry materials doped with RE (rare earth) ions, have been prepared (either single or double doped) and are extensively investigated due to their high luminescence. ${ }^{1-22}$ In the family of sulphates, $\mathrm{CaSO}_{4}$ phosphors, because of its high sensitivity, ease of preparation and stability of response in adverse climates, have already been very popular for use in radiation dosimetry, personnel dosimetry and environmental monitoring. ${ }^{23-25}$ In the presence of certain suitable impurities, particularly rare earth ions, $\mathrm{CaSO}_{4}$ phosphor shows efficient thermoluminescence. There are many works on TL, ESR, PL and various display applications of $\mathrm{CaSO}_{4}: \mathrm{RE}^{26-33}$

One of the most important among these is $\mathrm{CaSO}_{4}$ activated with $\mathrm{Dy}^{3+} . \mathrm{CaSO}_{4}$ :Dy is a unique inorganic phosphor material used in plasma dis- play panels. ${ }^{26}$ Another phosphor in $\mathrm{CaSO}_{4}$ series, which has been widely studied, is $\mathrm{CaSO}_{4}: \mathrm{Eu}^{34-36}$ Out of the different factors influencing the characteristics of TL phosphors, annealing is one of the most effective one. Many researchers reported that pre and post-irradiation annealing affects the glow curve structure and fading pattern of TL phosphors. Many researchers observed changes in TL glow curve structure of $\mathrm{CaSO}_{4}: \mathrm{Dy}$ phosphor with the increase in annealing temperature. ${ }^{37,38}$ Till now no paper is published on TL of $\mathrm{CaSO}_{4}$ doped with both Eu and Dy. In this paper the effect of annealing on the TL glow curve structure and fading of the prepared $\mathrm{Ca}_{0.99} \mathrm{SO}_{4}: \mathrm{Eu}_{0.005} \mathrm{Dy}_{0.005}$ phophor is reported.

\section{Materials and Methods}

\section{Method of preparation}

Different phosphors of $\mathrm{Ca}_{1-\mathrm{x}-\mathrm{y}} \mathrm{SO}_{4}: \mathrm{Eu}_{\mathrm{x}}, \mathrm{Dy}_{\mathrm{y}}(0<$ 
$\mathrm{x}<0.04,0<\mathrm{y}<0.04)$ were prepared by taking 1 $\mathrm{gm}$ of analytical reagent (AR) calcium chloride $\left(\mathrm{CaCl}_{2} .2 \mathrm{H}_{2} \mathrm{O}\right)$ and different amounts of (AR) europium oxide $\left(\mathrm{Eu}_{2} \mathrm{O}_{3}\right)$ and dysprosium chloride $\left(\right.$ DyCl $l_{3} \cdot 6 \mathrm{H}_{2} \mathrm{O}$ ). In this method $\mathrm{Eu}_{2} \mathrm{O}_{3}$ is dissolved in $\mathrm{AR}$ hydrochloric acid $(\mathrm{HCl})$ and then mixed with $\mathrm{CaCl}_{2} .2 \mathrm{H}_{2} \mathrm{O}$ and $\mathrm{DyCl}_{3} .6 \mathrm{H}_{2} \mathrm{O}$ which were dissolved in deionized water. Then, Ammonium sulphate $\left[\left(\mathrm{NH}_{4}\right)_{2} \mathrm{SO}_{4}\right]$ had been added to this solution in the presence of ethanol. $\left(\mathrm{NH}_{4}\right)_{2} \mathrm{SO}_{4}$ was added drop wise to the solution until the formation of precipitates were completed. The precipitates were separated and washed many times to remove the ethanol and kept in oven at $373 \mathrm{~K}$ for 2 hours. The dried precipitates were crushed and sieved to obtain fine mesh powders. The samples were further annealed at $873 \mathrm{~K}$ for 1 hour. The sample with $\mathrm{Eu}=0.5 \mathrm{at} \%$ and $\mathrm{Dy}=0.5$ at\% was found having the highest TL intensity and had been divided into several parts and subjected to annealing treatment at 873, 973, 1073 and $1173 \mathrm{~K}$ for 1 hour.

\section{Characterization}

The identification of phases in the synthesized compounds of $\mathrm{Ca}_{1-\mathrm{x} y} \mathrm{SO}_{4}: \mathrm{Eu}_{\mathrm{x}}, \mathrm{Dy}_{\mathrm{y}}(0<\mathrm{x}<$ $0.04,0<\mathrm{y}<0.04$ ) had been carried out by $\mathrm{X}$-ray diffraction studies using PAN alytical X-ray diffractometer with $\mathrm{Cu}-\mathrm{K} \alpha_{1}(\lambda=1.5406 \AA)$ radia-

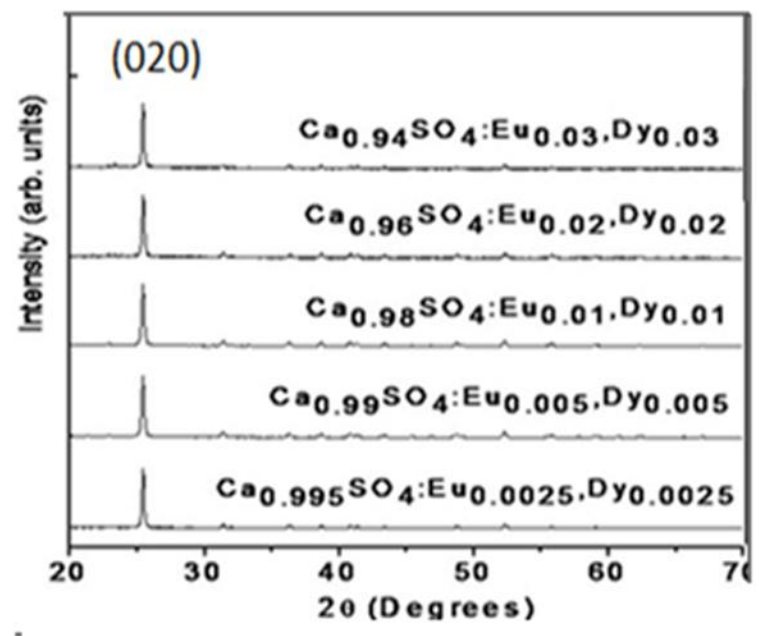

Figure 1 | XRD patterns of $\mathrm{Ca}_{1-\mathrm{x}-\mathrm{S}} \mathrm{SO}_{4}: \mathrm{Eu}_{\mathrm{x}}, \mathrm{Dy}_{\mathrm{y}}(0<\mathrm{x}<$ $0.04,0<y<0.04)$ samples annealed at $873 \mathrm{~K}$. tion having Ni filter, operated at $40 \mathrm{kV}$ and 40 $\mathrm{mA}$ at a step size of $\Delta 2 \theta=0.02^{\circ}$ over the angular range $20^{\circ} \leq 2 \theta \leq 80^{\circ}$ and comparing the interplanar distances and intensity values with those of the corresponding standard peaks using JCPDS files.

The thermoluminescence glow curves of the samples were recorded with the instrument (model TL 1404) consisting of a kanthal strip heated directly using temperature programmer, a photomultiplier (RCA 931A PMT), a DC amplifier and a millivolt recorder. TL glow curves are recorded with linear heating rate of $2.17 \mathrm{~K} \mathrm{sec}^{-1}$, taking 2-5 mg sample in each measurement.

\section{Results and Discussion}

\section{$X$-ray diffraction study}

Figure 1 shows the XRD patterns of $\mathrm{Ca}_{1-\mathrm{x}-}$ ${ }_{y} \mathrm{SO}_{4}: \mathrm{Eu}_{\mathrm{x}}, \mathrm{Dy}_{\mathrm{y}}(0<\mathrm{x}<0.04,0<\mathrm{y}<0.04)$ samples annealed at $873 \mathrm{~K}$. The sharp diffraction peaks can be perfectly indexed to the highly purity and crystallinity of $\mathrm{CaSO}_{4}$. All the XRD patterns are fitted well with the orthorhombic structure of $\mathrm{CaSO}_{4}$ (JCPDS no. 80-0787). Fig. 2 shows the XRD patterns of $\mathrm{Ca}_{0.99} \mathrm{SO}_{4}: \mathrm{Eu}_{0.005}$, Dy $\mathrm{y}_{0.005}$ phosphor an-

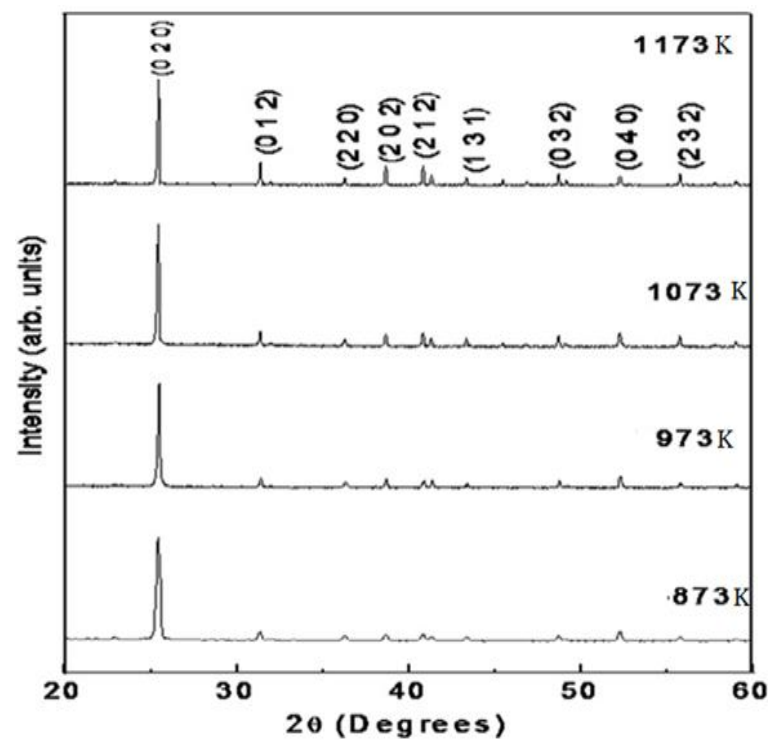

Figure 2 | XRD patterns of $\mathrm{Ca}_{0.99} \mathrm{SO}_{4}: \mathrm{Eu}_{0.005}, \mathrm{Dy}_{0.005}$ phosphor annealed at different temperatures. 


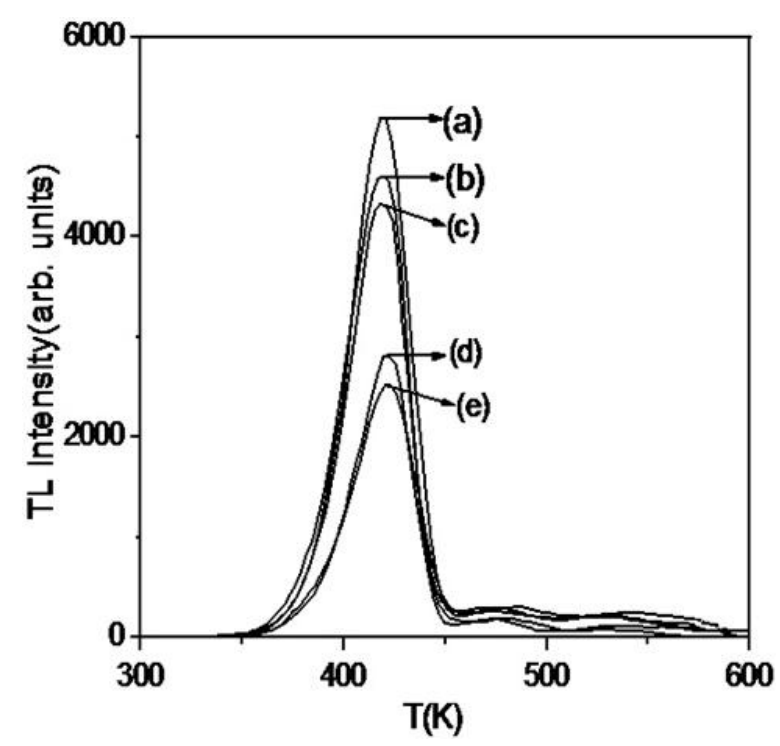

Figure 3 | TL glow curves of $\mathrm{Ca}_{1-x-y} \mathrm{SO}_{4}: \mathrm{Eu}_{x}, \mathrm{Dy}_{y}$, $(0<x<0.04,0<y<0.04)$ annealed at $873 \mathrm{~K}$ and irradiated with 150 Gy of $y$-ray [ $x, y=$ (a) 0.5 at\%, 0.5 at\%, (b) 0.25 at\%, 0.25 at\% (c) 1 at\%, 1 at\%, (d) 2 at\%, 2 at\%, (d) 3 at\%, 3 at\%].

nealed at different temperatures ranging from 873-1173 K for 1 hour.

\section{Thermoluminescence study}

Concentration effect. Figure 3 shows the TL glow curves of $\mathrm{Ca}_{1-\mathrm{x}-\mathrm{SO}} \mathrm{SO}_{4}: \mathrm{Eu}_{\mathrm{x}}, \mathrm{Dy}_{\mathrm{y}}(0<\mathrm{x}<0.04,0<$ $\mathrm{y}<0.04)$, annealed at $873 \mathrm{~K}$ and irradiated with $150 \mathrm{~Gy}$. It is observed from the figure that the phosphor $\mathrm{Ca}_{1-\mathrm{x}-\mathrm{y}} \mathrm{SO}_{4}: \mathrm{Eu}_{\mathrm{x}}, \mathrm{Dy}_{\mathrm{y}}$ has the highest $\mathrm{TL}$ intensity for $\mathrm{x}=0.5$ at $\%=0.005$ and $\mathrm{y}=0.5 \mathrm{at} \%=$ 0.005. All the TL glow curves have the same structure, having one prominent peak at around $420 \mathrm{~K}$ and two peaks at around 475 and $540 \mathrm{~K}$, the intensities of these glow peaks being very low compared to the prominent peak. The appearance of three peaks in the glow curves shows that three different sets of traps are being activated due to $\gamma$-irradiation for all concentrations of Eu and Dy within temperature range of 320-604 K. Fig. 4 shows the variation of TL intensity of the prominent peak at $420 \mathrm{~K}$ with change of sum of dopant concentrations $(\mathrm{x}+\mathrm{y})$. It is seen

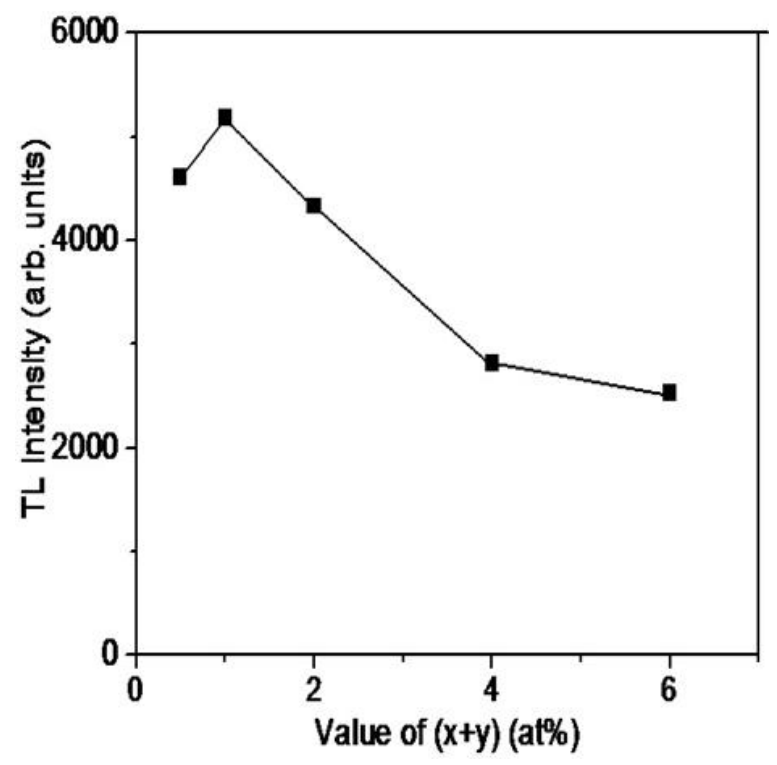

Figure 4 | TL intensity of the prominent peak at $420 \mathrm{~K}$ of $\mathrm{Ca}_{1-\mathrm{x}-\mathrm{y}} \mathrm{SO}_{4}: \mathrm{Eu}_{x}, \mathrm{Dy}_{\mathrm{y}}$ versus dopant concentrations $(x+y)$.

that each TL glow curve has one prominent peak at around $420 \mathrm{~K}$ and two shoulder peaks at around 475 and $540 \mathrm{~K}$.

Effect of pre-irradiation annealing temperature and duration on TL glow curve. Figure 5 shows the TL glow curves of $\mathrm{Ca}_{0.99} \mathrm{SO}_{4}: \mathrm{Eu}_{0.005} \mathrm{Dy}_{0.005}$ annealed at different temperatures and irradiated with $150 \mathrm{~Gy}$ of $\mathrm{\gamma}$-rays. The glow curve exhibits a dosimetric peak $\mathrm{P}_{1}$ at $420 \mathrm{~K}$ and two very low intensity peaks $\mathrm{P}_{2}$ and $\mathrm{P}_{3}$ at around 485 and $540 \mathrm{~K}$ respectively. As shown in fig., there is no drastic change in the glow curve structure with the annealing temperature. Table 1 shows the relative TL intensities of the peaks and the corresponding peak height ratio (dosimetric peak to high temperature peaks). As the annealing temperature increases, intensities of peaks also increase. But the TL intensity of the peak at $420 \mathrm{~K}$ for the annealing temperature $973 \mathrm{~K}$ is almost same with the $1073 \mathrm{~K}$ which shows the crystal/defect structure stability of $\mathrm{CaSO}_{4}: \mathrm{Eu}$, Dy even at such a high temperature. Bakshi et al. ${ }^{37,38}$ observed con- 
siderable change in TL glow curve structure of $\mathrm{CaSO}_{4}$ :Dy with post preparation annealing in the temperature range $300-850^{\circ} \mathrm{C}$ and $650-1000^{\circ} \mathrm{C}$. With increasing annealing temperature, intensity of dosimetric peak at $240^{\circ} \mathrm{C}$ reduces, whereas the low temperature satellite peak increases. Their study indicates that post irradiation annealing of this phosphor should be carried out in the temperature range $650-700^{\circ} \mathrm{C}$ in order to maintain the dosimetric properties for further application in radiation dosimetry.

To check effect annealing duration on glow curve structure as well as TL intensity, $\mathrm{Ca}_{0.99} \mathrm{SO}_{4}: \mathrm{Ey}_{0.005} \mathrm{Dy}_{0.005}$ phosphor has been annealed at $700^{\circ} \mathrm{C}$ for 1,3 and 4 hours. The recorded glow curves are shown in Figure 6. TL intensity of the prominent peak decreases with annealing duration. For the annealing duration of 4 hours, the glow curve becomes more complex, appearing one more peak in the lower temperature side $(\approx 376 \mathrm{~K})$.

To check the effect of annealing on fading of $\mathrm{Ca}_{99} \mathrm{SO}_{4}: \mathrm{Ey}_{.005} \mathrm{Dy} .005$ phosphor, samples annealed at different temperatures were stored at room temperature for different durations. Figure 7 (a) shows the fading pattern of peak $\mathrm{P}_{1}$ at $\mathrm{Tm}=420$ $\mathrm{K}$ in phosphor annealed at $873 \mathrm{~K}$. TL intensity reduces by $20 \%$ in four days, which remains constant up to $7^{\text {th }}$ day of storage. After that the intensity is further decreased by $27 \%$ and then remains constant during experiments. As shown in Figure 7 (b), the fading of the sample annealed at $973 \mathrm{~K}$ is very fast. TL intensity reduces by $40 \%$ in 7 days The sample annealed at $1073 \mathrm{~K}$ shows $34 \%$ fading in 4 days remains constant for 4 days, after which it fades very fast (Fig. 7 (c)).

\section{Conclusion}

The newly preparedCaSO ${ }_{4}$ phosphor activated with different concentrations of Eu and Dy have its maximum TL intensity for $\mathrm{Eu}=0.5$ at\% and Dy $=0.5$ at $\%$. XRD pattern shows that the phosphors have orthorhombic structure (JCPDS NO. 800787). The effects of annealing temperature on the TL glow curves have been discussed. Unlike $\mathrm{CaSO}_{4}$ :Dy, as reported by many researchers, there is no considerable change in the glow curve structure of $\mathrm{CaSO}_{4}$ : $\mathrm{Eu}$,Dy. The sample annealed at $1173 \mathrm{~K}$ has the highest TL intensity. If the annealing duration increases the TL glow curve becomes more complex, i.e. peaks in the lower temperature side appears.

\section{References}

I. Deben, M.S., Rangeela, Y.D. \& Dorendrajit, S.S. (2009). Effect of annealing temperature on thermoluminescence glow curve of X-irradiated $\mathrm{BaSO}_{4}: \mathrm{Pr}$. Indian Journal of Pure and Apllied Physics 47, 432-434

2. Rangeela, Y.D. \& Dorendrajit, S.S. (2012). Synthesis and TL glow curve analysis of Thermoluminescence (TL) characteristics of $\mathrm{BaSO}_{4}: \mathrm{Eu}, \mathrm{Dy}$ phosphor. Journal of Luminescence 132, $1575-1580$.

3. Azorin, J., Furetta, C. \& Scacco, A. (1993). Preparation and properties of thermoluminescent materials. Physica Status Solidi Ar38, 9

4. Manam, J. \& Das, S. (2010). Characterization and TSL dosimetric properties of $\mathrm{Mn}$ doped $\mathrm{BaSO}_{4}$ phosphor prepared by recrystallisation method. Journal of Alloys and Compound 489, 84-90

5. Yamashita, N.,Yamamoto, I., Ninagawa, K.,Wada, T.,Yamashita, Y. \& Nakao, Y. (1985). photoluminescence and thermoluminescence of $\mathrm{MgSO}_{4}, \mathrm{CaSO}_{4}$, $\mathrm{SrSO}_{4}$ and $\mathrm{BaSO}_{4}$ powder phosphors activated with $\mathrm{Tb}^{3}$. Japanese Journal of Applied Physics 24, $\mathrm{II} 74$

6. Ajorin, J.N. \& Rubio, N.O. (1994). Optical absorption and thermoluminescence of $\mathrm{D}^{3+}$ doped $\mathrm{BaSO}_{4}$ gamma irradiated at room temperature. Journal of Physics: Condensed Matter 6, 383I-3837.

7. GunduRao, T.K., Shinde, S.S., Bhattt, B.C., Srivastava, J.K. \& Nambi, K.S.V. (1995). Electron spin resonance, thermoluminescence and fluorescence studies on Ba$\mathrm{SO}_{4}: \mathrm{Eu}$ and $\mathrm{BaSo}$ :Eu, $\mathrm{P}$ thermoluminescent phosphors. Journal of Physics: Condensed Matter 7 , 6569458I.

8. Manam, J., Puja, K. \& Das, S. (2OII). Characterization and photoluminescence studies of Eu2+-doped $\mathrm{BaSO}_{4}$ phosphor prepared by the recrystallization method. Applied Physics $A$ 104, 197-203

9. Anant, P., Kanika, S.R., Shaila B. \& Birendra, S. (2017). Nanocrystalline Europium doped barium sulphate as an energy independent thermoluminescent dosimeter. AIP Conference Proceedings, I832:050034

Io. Luthra, J.M. \& Narendra, G.M. (1974). Mechanism of thermoluminescence in Sm- and Eu-doped barium sul- 
phate. Journal of Luminescence 9, 94-IO3

II. Rohitash, S., Dhasmana, M.K. \& Rawat, R.B.S. (2013). Thermoluminescence of nano-crystalline $\mathrm{Eu}$ doped $\mathrm{BaSO}_{4}$.International Journal of Engineering and Science 2, I6-19

I2. Gupta, N.M., Luthra, J.M. \& Shankar, J. (1974).Trapping and emission centres in thermoluminescent barium sulphate. Radiation effects 21,15I-I56

13. Matthews, R.J. \& Stoebe, T.G. (1982). Thermoluminescent spectra and optical absorption in $\mathrm{CaSO}_{4}: \mathrm{Dy}$. Jour nal of Physics C: Solid State Physics 15, 627I

I4. Nagpal, J.S. \& Varadharajan, G. (1982). Thermoluminescence of rare earth doped $\mathrm{BaSO}_{4}$ phosphors and its applications. The International Journal of Applied Radiation and Isotopes 33, 175-182

I5. Manam, J. \& Das, S. (2009). Determination of kinetic parameters of thermally stimulated luminescence of $\mathrm{Cu}$ doped $\mathrm{BaSO}_{4}$. Journal of Physics and Chemistry of Solids 70, 379-384

16. Kamarudin, N. (2014). Thermoluminescence (TL) properties and $\mathrm{x}$-ray diffraction $(\mathrm{XRD})$ analysis of high purity $\mathrm{CaSO}_{4}$ :Dy TL material. AIP Conference Proceedings 1614, 94

17. Kasa, I., Chobola, R., Mell, P., Szakacs, S. \& Kerekes, A. (2007). Preparation and investigation of thermoluminescence properties of $\mathrm{CaSO}_{4}: \mathrm{Tm}, \mathrm{Cu}$. Radiation Protection Dosimetry 123, 32-35,

I8. Wani, J.A., Atone, M.S., \& Dhoble, S.J. (2013). The influence of different synthesis routes on thermoluminescence of $\mathrm{CaSO}_{4}$ : Dy, P, Ce phosphors. Advanced Material Letters 4, 363-367

19. Salah, N., Sahare, P.D., Lochab, S.P. \& Kumar, P. (2006).TL and PL studies on $\mathrm{CaSO}_{4}$ : Dy nanoparticles.Radiation Measurement 4I, 40-47

2o. Nagpal, J.S. \& Varadharajan, G. (1982). Thermoluminescence of rare earth doped $\mathrm{BaSO}_{4}$ phosphors and its applications. The International Journal of Applied Ra- diation and Isotopes 33, 175-182

2I. Manam, J. \& Das, S. (2009). Determination of kinetic parameters of thermally stimulated luminescence of $\mathrm{Cu}$ doped $\mathrm{BaSO}_{4}$. J. Physics and Chemistry of Solids 7o, 379 $-384$

22. Lakshmanan, A.R. (1999). Photoluminescence and thermoluminescence processes in rare earth doped $\mathrm{CaSO}_{4}$ phosphors. Progress in Materials Science 44, I-I87

23. Kim, J.L., Chang, S.Y. \& Kim, B.H. (1999). IAEA/RCA Personal dosemeter intercomparison results for the KAERI TLD system. Radiation Protection Dosimetry 85, I53-157.

24. Vohra, K.G., Bhatt, R.C., Bhuwan, C., Pradhan, A.S., Lakshmanan, A.R. \& Shashtry, M. S.S. (1980). A personnel dosimeter TLD badge based on $\mathrm{CaSO}_{4}$ : Dyteflon TLD discs. Health Phyics 38, 193-197.

25. Townsend, P.D. \& White, D.R. (1996). Thermoluminescence Emission, Excitation and Stimulation Spectra of $\mathrm{CaSO}_{4}:$ Dy and $\mathrm{CaSO}_{4}:$ Tm. Radiation Protection Dosimetry 65, 281.

26. Kolk, E.V., Dorenbos, P., Vink, A.P., Perego, R.C., Van, C.W.E. \& Lahshmanan, A.R (200I). Emission spectrum of $\mathrm{Pr}^{3+}$ recorded at $293 \mathrm{~K}$ of $\mathrm{CaSo}_{4}: \mathrm{Pr}^{3+}$ (o.2\%) at $160 \mathrm{~nm}$. Physics Review B 64, $195 \mathrm{I29}$.

27. Kamarudin, N. (2014). Thermoluminescence (TL) properties and $\mathrm{x}$-ray diffraction (XRD) analysis of high purity $\mathrm{CaSO}_{4}$ :Dy TL material. AIP Conference Proceedings 1614, 94.

28. Sanyal, B., Natarajan, V., Chawla, S. P. \& Sharma, A. (20I0). TL and EPR studies of $\mathrm{CaSO}_{4}$ :Dy phosphor to investigate its efficacy in measurement of food irradiation dose at sub-ambient temperatures. Radiation Measurement 45, 899-905. 